\title{
Turing Bifurcation and Pattern Formation of Stochastic Reaction-Diffusion System
}

\author{
Qianiqian Zheng, ${ }^{1}$ Zhijie Wang, ${ }^{1}$ Jianwei Shen, ${ }^{2}$ and Hussain Muhammad Ather Iqbal ${ }^{1}$ \\ ${ }^{1}$ College of Information Science and Technology, Donghua University, Shanghai 201620, China \\ ${ }^{2}$ Institute of Applied Mathematics, Xuchang University, Xuchang, Henan 461000, China \\ Correspondence should be addressed to Zhijie Wang; wangzj@dhu.edu.cn and Jianwei Shen; xcjwshen@gmail.com
}

Received 28 August 2016; Revised 18 November 2016; Accepted 15 December 2016; Published 12 February 2017

Academic Editor: Zhi-Yuan Sun

Copyright ( 2017 Qianiqian Zheng et al. This is an open access article distributed under the Creative Commons Attribution License, which permits unrestricted use, distribution, and reproduction in any medium, provided the original work is properly cited.

\begin{abstract}
Noise is ubiquitous in a system and can induce some spontaneous pattern formations on a spatially homogeneous domain. In comparison to the Reaction-Diffusion System (RDS), Stochastic Reaction-Diffusion System (SRDS) is more complex and it is very difficult to deal with the noise function. In this paper, we have presented a method to solve it and obtained the conditions of how the Turing bifurcation and Hopf bifurcation arise through linear stability analysis of local equilibrium. In addition, we have developed the amplitude equation with a pair of wave vector by using Taylor series expansion, multiscaling, and further expansion in powers of small parameter. Our analysis facilitates finding regions of bifurcations and understanding the pattern formation mechanism of SRDS. Finally, the simulation shows that the analytical results agree with numerical simulation.
\end{abstract}

\section{Introduction}

The pattern formation was first investigated and interpreted by Turing sixty years ago [1]. Othmer and Scriven [2] proposed that the Turing instability which is initially stable steady-state of a dynamical system can become unstable if we consider diffusion in the system. It is also possible in networkorganized systems which is important for understanding of multicellular morphogenesis. Recently Turing bifurcation, amplitude equation, and secondary bifurcation have become more significant to study the pattern formation [3-5]; Lee and Cho found that the shape and type of Turing patterns depend on dynamical parameters and external periodic forcing [6]. Moreover, Peña and Pérez-García showed that slightly squeezed hexagons are locally stable in a full range of distorted angles [7]. The domain coarsening process is strongly affected by the spatial separation between groups created by the Turing pattern formation process [8] and the robustness problem is also investigated [9]. The effects of cross-diffusion, the phenomenon in which a gradient in the concentration of one species induces the change of other species, on pattern formation in Reaction-Diffusion Systems have been discussed in many theoretical papers [10]. Fanelli et al. [11] showed that cross-diffusion can destabilize uniform equilibrium which is stable for the kinetic and self-diffusion reaction systems. On the other hand, cross-diffusion can also stabilize a uniform equilibrium which is stable for the kinetic system but unstable for the self-diffusion reaction system [12]. In conclusion, spatial patterns in Reaction-Diffusion Systems have attracted the interest of experimentalists and theorists during the last few decades. However, until now, no general theoretical analysis has been proposed for the possible role of noise in dissipative pattern formation.

Noise is a ubiquitous phenomenon in nature and is always deemed to play a very important role in natural synthetic system [13]. Coherence resonance and stochastic resonance in a noise-driven gene network regulated by small RNA $[14,15]$. Viney and Reece [16] treated noise as adaptive and suggested that applying evolutionary rigour to the study of noise is necessary to fully understand organismal phenotypes. Scarsoglio et al. [17] presented different stochastic mechanisms of spatial pattern formation with a variable as noise-induced phenomena. Hori and Hara provided a mechanistic basis of Turing pattern formation that is induced by intrinsic noise 
and derived an efficient computation tool to examine the spatial power spectrum of the intrinsic noise [18]. Sun et al. [19] revealed that noise can make the regular circle pattern to be a target wave-like pattern by numerical simulations. A stochastic version of the Brusselator model is proposed and studied via the system size expansion [20] and the mesoscopic equations governing the dynamics were derived and used to special models [21]. Many studies have been presented in these research areas [22-28], as practice shows that theory on Turing bifurcation and pattern formation in dynamical system was rarely studied.

It is known that amplitude equation is not only a promising tool to investigate the RDS but also the main focus of the pattern dynamics $[29,30]$. However, the amplitude equation is a complex process [31], and only a few systems have been chosen in the past for amplitude equation [32-35]. In this paper, we studied pattern selection of amplitude equation with a pair of wave vector by using the standard multiple scale analysis $[36,37]$. Previously, the researchers did not take into account the effect of noise when deriving the amplitude equation but we will include it.

Besides the study of patterns, it can offer useful information on the underlying processes causing possible changes in the system. In order to better understand the reaction diffusion model, first, we proposed to study the pattern formation with noise based on the theory. In this paper, we obtained some interesting results explaining biological mechanism in a modified system. Moreover, we also investigated the relationship between the Reaction-Diffusion System and noise, revealing how the dynamics of the model regulation is affected by noise which provides a way to investigate the mechanism of pattern formation.

The paper is organized as follows. In Section 2, we present the general reaction diffusion with noise and derive the condition of Hopf bifurcation and Turing bifurcation. In Section 3, we derive the amplitude equation from ReactionDiffusion System with noise. In Section 4, we utilize an example to illustrate the application of these ideas and using simulations validate theoretical results and present some interesting pattern dynamical phenomena. Finally, we summarize our results and conclude.

\section{Turing Bifurcation with SRDS}

Since we know that noise plays an important role in the nonlinear systems, some promising results have been presented $[11,12,32]$. However, most people investigated noise by simulation and seldom put forward the theoretical conclusion, especially on pattern formation. In this paper, we study the effect of noise on pattern formation by deriving the Turing bifurcation, to know how it affects the pattern formation. The general diffusion form with noise is as follows:

$$
\begin{aligned}
& \frac{\partial u}{\partial t}=f(u, v)+d_{1} \nabla^{2} u+d_{2} R_{1}(u, v) \xi_{1}, \\
& \frac{\partial v}{\partial t}=g(u, v)+d_{3} \nabla^{2} v+d_{4} R_{2}(u, v) \xi_{2}
\end{aligned}
$$

where $\xi$ is the noise and $\nabla^{2}$ is the Laplace operator; $d_{1}, d_{3}$ and $d_{2}, d_{4}$ are diffusion parameters and noise magnitude, respectively.

For convenience, we just consider $R_{1}(u, v)=u-u_{0}$, $R_{2}(u, v)=v-v_{0}, \xi_{1}=(1 / \sqrt{2 \pi} \sigma) e^{-\left(u-u_{0}\right)^{2} / 2 \sigma^{2}}, \xi_{2}=(1 /$ $\sqrt{2 \pi} \sigma) e^{-\left(\nu-v_{0}\right)^{2} / 2 \sigma^{2}}$ as random variable in this system. In order to obtain the stability of this spatially uniform solution, we consider a perturbation of the form in the following:

$$
P(t)=\left(\begin{array}{l}
u(t) \\
v(t)
\end{array}\right)=\left(\begin{array}{c}
u(t)-u_{0} \\
v(t)-v_{0}
\end{array}\right) .
$$

In the convergence domain, we can obtain the linear system of stochastic system as (3) at $\left(u_{0}, v_{0}\right)$ which satisfy $f\left(u_{0}, v_{0}\right)=0, g\left(u_{0}, v_{0}\right)=0$.

$$
\begin{aligned}
& \frac{\partial u}{\partial t}=a_{11} u+a_{12} v+d_{1} \nabla^{2} u+d_{21} u, \\
& \frac{\partial v}{\partial t}=a_{21} u+a_{22} v+d_{3} \nabla^{2} v+d_{41} v,
\end{aligned}
$$

where the matrix $a$ is the partial derivative of $f(u, v), g(u, v)$ at $\left(u_{0}, v_{0}\right)$ and $d_{21}=d_{2}(1 / \sqrt{2 \pi} \sigma), d_{41}=d_{4}(1 / \sqrt{2 \pi} \sigma)$.

For convenience, we can get the linearized system governing the dynamics of $P$ is defined by

$$
P_{t}=A P+D \Delta P,
$$

where the coefficient matrix is given by

$$
\begin{aligned}
& A=\left(\begin{array}{ll}
A_{11} & A_{12} \\
A_{21} & A_{22}
\end{array}\right), \\
& D=\left(\begin{array}{ll}
d_{1} & 0 \\
0 & d_{3}
\end{array}\right),
\end{aligned}
$$

where

$$
\begin{aligned}
& \mathrm{A}_{11}=a_{11}+d_{21}, \\
& A_{12}=a_{12}, \\
& A_{22}=a_{22}+d_{41}, \\
& A_{21}=a_{21} .
\end{aligned}
$$

In the standard way, we assume that $P$ take the form as

$$
P=\left(\begin{array}{c}
c_{1} \\
c_{2}
\end{array}\right) e^{\lambda t+i k r}
$$

and get the characteristic equation from system (4) as follows:

$$
\left|\begin{array}{cc}
\lambda_{k}-A_{11}+k^{2} d_{1} & -A_{12} \\
-A_{21} & \lambda_{k}-A_{22}+k^{2} d_{3}
\end{array}\right|=0 .
$$

Finally, we solve the characteristic equation and obtain the eigenvalues

$$
\begin{aligned}
\lambda_{k}^{2}-\operatorname{Tr}_{k} \lambda+\delta\left(k^{2}\right) & =0 \\
\lambda_{k} & =\frac{1}{2}\left(\operatorname{Tr}_{k} \pm \sqrt{\operatorname{Tr}_{k}^{2}-4 \delta_{k}}\right),
\end{aligned}
$$


where

$$
\begin{aligned}
\operatorname{Tr}_{k}= & A_{11}+A_{22}-k^{2}\left(d_{1}+d_{3}\right) \\
= & a_{11}+d_{21}+a_{22}+d_{41}-k^{2}\left(d_{1}+d_{3}\right), \\
\operatorname{Tr}_{0}= & A_{11}+A_{22}=a_{11}+d_{21}+a_{22}+d_{41} \\
\delta\left(k^{2}\right)= & A_{11} A_{22}-A_{12} A_{21}-\left(A_{11} d_{3}+A_{22} d_{1}\right) k^{2} \\
& +d_{1} d_{3} k^{4} \\
= & a_{11} a_{22}+a_{11} d_{41}+d_{21} a_{22}+d_{21} d_{41}-a_{12} a_{21} \\
& -\left(a_{11} d_{3}+d_{21} d_{3}+a_{22} d_{1}+d_{1} d_{41}\right) k^{2} \\
& +d_{1} d_{3} k^{4} \\
\operatorname{det}(P)= & \delta_{0} \\
= & a_{11} a_{22}+a_{11} d_{41}+d_{21} a_{22}+d_{21} d_{41}-a_{12} a_{21} .
\end{aligned}
$$

Based on the bifurcation theory, we obtain new conditions of bifurcation with noise.

(1) Hopf bifurcation occurs in the Reaction-Diffusion System (3) which should satisfy the following critical conditions here:

(i) $a_{11}+d_{21}+a_{22}+d_{41}=0$,

(ii) $a_{11} a_{22}+a_{11} d_{41}+d_{21} a_{22}+d_{21} d_{41}-a_{12} a_{21}>0$,

(iii) $\operatorname{Re}\left(\lambda_{k}\right)$ is not a constant.

(2) Turing bifurcation (diffusion-driven instability) occurs in the Reaction-Diffusion System (3) which should satisfy the following conditions here:

(i) $a_{11}+d_{21}+a_{22}+d_{41}<0$,

(ii) $a_{11} a_{22}+a_{11} d_{41}+d_{21} a_{22}+d_{21} d_{41}-a_{12} a_{21}>0$,

(iii) $a_{11} d_{3}+d_{21} d_{3}+a_{22} d_{1}+d_{41} d_{1}>0$,

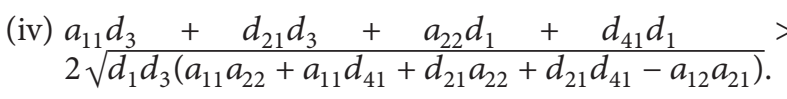

And the critical condition where $k \neq 0$ is

$$
\begin{aligned}
a_{11} a_{22} & +a_{11} d_{41}+d_{21} a_{22}+d_{21} d_{41}-a_{12} a_{21} \\
- & \left(a_{11} d_{3}+d_{21} d_{3}+a_{22} d_{1}+d_{1} d_{41}\right) k^{2}+d_{1} d_{3} k^{4}
\end{aligned}
$$

$=0$.

\section{Amplitude Equation with a Pair of Wave Vector}

For a modified model [38] with the external stimulus $\gamma u v$, the following is obtained:

$$
\begin{aligned}
& \frac{\partial u}{\partial t}=u-u^{3}-\alpha v-\gamma u v+d_{1} \nabla^{2} u+d_{2} R_{1}(u, v) \xi_{1} \\
& \frac{\partial v}{\partial t}=u-\beta v+d_{3} \nabla^{2} v+d_{4} R_{2}(u, v) \xi_{2} .
\end{aligned}
$$

In this paper, we expanded (12) at equilibrium $(0,0)$ by using the Taylor expansion and then we truncated the expansion at third order; it is found that only third order $u e^{-u^{2}}=u-u^{3}+\cdots$ will be included and higher order will not affect the amplitude equation in the process. And it can be written as

$$
\begin{aligned}
& \frac{\partial u}{\partial t}=u-u^{3}-\alpha v-\gamma u v+d_{21} u-d_{22} u^{3}+d_{1} \nabla^{2} u, \\
& \frac{\partial v}{\partial t}=u-\beta v+d_{41} v-d_{42} v^{3}+d_{3} \nabla^{2} v .
\end{aligned}
$$

In the following, we use multiple scale analysis to derive the amplitude equations with a pair of wave vector when $|k|=k_{c}$. Denote $\beta$ as the controlled parameters. When the controlled parameter is larger than the critical value of Turing point, the solutions of the systems (13) can be expanded as

$$
c=c_{0}+Z \mathrm{e}^{i k_{c} r}+\bar{Z} e^{-i k_{c} r} .
$$

Close to onset $\beta=\beta_{c}$, one has that $\partial Z / \partial t=s Z+F(Z)$.

Based on the center manifold near the Turing bifurcation point, it can be concluded that amplitude $Z$ satisfies $\partial Z / \partial t=$ $F(Z, \bar{Z})$.

From the standard multiple scale analysis, up to the third order in the perturbations, the spatiotemporal evolution of the amplitudes can be described as

$$
\begin{aligned}
\tau_{0} \frac{\partial Z}{\partial t}= & \mu Z+b \bar{Z}+c Z^{2}+d|Z|^{2}+e \bar{Z}^{2}+f Z^{3} \\
& +g|Z|^{2} Z+h|Z|^{2} \bar{Z}+i \bar{Z}^{3}+O\left(Z^{4}\right) .
\end{aligned}
$$

Due to spatial translational symmetry, we have the following equation:

$$
\begin{aligned}
\tau_{0} e^{i \phi} \frac{\partial Z}{\partial t}= & e^{i \phi} \mu Z+e^{-i \phi} b \bar{Z}+e^{i 2 \phi} c Z^{2}+d|Z|^{2} \\
& +e^{-i 2 \phi} e \bar{Z}^{2}+e^{i 3 \phi} f Z^{3}+e^{i \phi} g|Z|^{2} Z \\
& +e^{-i \phi} h|Z|^{2} \bar{Z}+e^{-i 3 \phi} i \bar{Z}^{3}+O\left(Z^{4}\right) .
\end{aligned}
$$

Comparing (15) with (16) and from the center manifold theory, we know that amplitude equation does not include the amplitude with unstable mode. As a result, we have the following equations:

$$
\tau_{0} \frac{\partial Z}{\partial t}=\mu Z-g|Z|^{2} Z .
$$

In the following, we will give the expressions of $\tau_{0}, \mu$, and $g$. Let system (13) be written as

$$
\frac{\partial c}{\partial t}=L c+N(c, c)
$$

where

$$
c=\left(\begin{array}{l}
u \\
v
\end{array}\right)
$$


is the variable,

$$
L=\left(\begin{array}{cc}
d_{21}+1+d_{1} \nabla^{2} & -\alpha \\
1 & d_{41}-\beta+d_{3} \nabla^{2}
\end{array}\right)
$$

is the linear operator, and

$$
N=\left(\begin{array}{c}
\gamma u v-u^{3}-d_{22} u^{3} \\
-d_{42} v^{3}
\end{array}\right)
$$

is the nonlinear term, where $d_{22}=\left(1 / 2 \sigma^{2}\right) d_{21}$ and $d_{42}=$ $\left(1 / 2 \sigma^{2}\right) d_{41}$.

We need to investigate the dynamical behavior when $\beta$ is close to $\beta_{c}$, and then we expand $\beta$ as

$$
\beta_{c}-\beta=\varepsilon \beta_{1}+\varepsilon^{2} \beta_{2}+\cdots,
$$

where $\varepsilon$ is a small enough parameter. We expand $c$ and $N$ as the series form of $\varepsilon$ :

$$
c=\left(\begin{array}{l}
u_{1} \\
v_{1}
\end{array}\right) \varepsilon+\left(\begin{array}{l}
u_{2} \\
v_{2}
\end{array}\right) \varepsilon^{2}+\cdots
$$

and $N$ in the Appendix.

Linear operator $L$ can be expanded as

$$
L=L_{c}+\left(\gamma_{c}-\gamma\right) M
$$

and $L_{c}$ and $M$ in the Appendix.

Let

$$
\begin{gathered}
T_{0}=t, \\
T_{1}=\varepsilon t, \\
T_{2}=\varepsilon^{2} t,
\end{gathered}
$$

and $T_{i}$ is a dependent variable. For the derivation of time, we have that

$$
\frac{\partial}{\partial t}=\varepsilon \frac{\partial}{\partial T_{1}}+\varepsilon^{2} \frac{\partial}{\partial T_{2}}+\cdots
$$

The solutions of systems (13) have the following form:

$$
c=\left(\begin{array}{l}
u \\
v
\end{array}\right)=\left(\begin{array}{l}
x \\
y
\end{array}\right) e^{i k_{c} r}+c . c .
$$

This expression implies that the bases of the solutions have nothing to do with time and the amplitude $W$ is a variable that changes slowly. As a result, it can be written generally as the following equation:

$$
\frac{\partial W}{\partial t}=\varepsilon \frac{\partial W}{\partial T_{1}}+\varepsilon^{2} \frac{\partial W}{\partial T_{2}}+\cdots
$$

Substituting the above equations into (24) and expanding (24) according to different orders of $\varepsilon$, we can obtain three equations as follows:

$$
\begin{aligned}
& \varepsilon: L_{c}\left(\begin{array}{l}
u_{1} \\
v_{1}
\end{array}\right)=0, \\
& \varepsilon^{2}: L_{c}\left(\begin{array}{l}
u_{2} \\
v_{2}
\end{array}\right)=N_{1}, \\
& \varepsilon^{3}: L_{c}\left(\begin{array}{l}
u_{3} \\
v_{3}
\end{array}\right)=N_{2}
\end{aligned}
$$

and $N_{1}, N_{2}$ in the Appendix.

We first consider the case of the first order of $\varepsilon$. Since $L_{c}$ is the linear operator of the system close to the onset, $\left(u_{1}, v_{1}\right)^{T}$ is the linear combination of the eigenvectors that corresponds to the zero eigenvalue since that

$$
\left(\begin{array}{l}
u_{1} \\
v_{1}
\end{array}\right)=\left(\begin{array}{l}
x_{1} \\
y_{1}
\end{array}\right) W e^{i k_{c} r}+\text { c.c. }
$$

Let $x_{1}=p$ by assuming $y_{1}=1$; then,

$$
\left(\begin{array}{l}
u_{1} \\
v_{1}
\end{array}\right)=\left(\begin{array}{l}
p \\
1
\end{array}\right)\left(W e^{i k_{c} r}+c . c .\right),
$$

where $p$ in the Appendix and $W$ is the amplitude of the mode $e^{i k_{c} r}$.

Now, we consider the case of the second order of $\varepsilon^{2}$. According to the Fredholm solubility condition [32], the vector function of the right hand of the above equation must be orthogonal with the zero eigenvectors of operator $L_{c}^{+}$. And the zero eigenvectors of adjoint operator $L_{c}^{+}$are

$$
\left(\begin{array}{l}
1 \\
q
\end{array}\right) e^{-i k_{c} r}
$$

and $q$ in the Appendix.

It can be obtained from the orthogonality condition that

$$
\tau_{0} \frac{\partial}{\partial T_{1}}(W)=\beta_{1} W .
$$

By using the same methods, we deduce

$$
\left(\begin{array}{l}
u_{2} \\
v_{2}
\end{array}\right)=\left(\begin{array}{l}
a_{0} \\
b_{0}
\end{array}\right)+\left(\begin{array}{l}
a_{1} \\
b_{1}
\end{array}\right) e^{i k_{c} r}+\left(\begin{array}{l}
a_{i i} \\
b_{i i}
\end{array}\right) e^{i 2 k_{c} r}
$$

and coefficients in the Appendix.

For the case of the third order of $\varepsilon^{3}$, replace $u_{1}, v_{1}, u_{2}$, and $v_{2}$ by their expression

$$
L_{c}\left(\begin{array}{l}
u_{2} \\
v_{2}
\end{array}\right)=B=C
$$

and $B, C$ in the Appendix. Using the Fredholm solubility condition again, we can obtain

$$
\tau_{0} \frac{\partial b_{1}}{\partial T_{1}}+\tau_{0} \frac{\partial W}{\partial T_{2}}=\beta_{1} v_{2}+\beta_{2} v_{1}-g|W|^{2} W .
$$




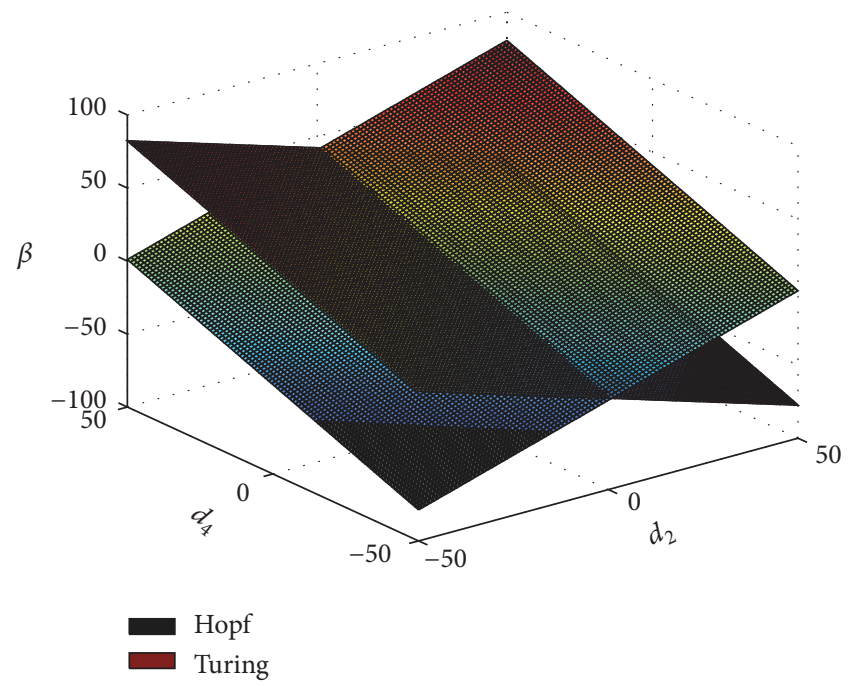

(a)

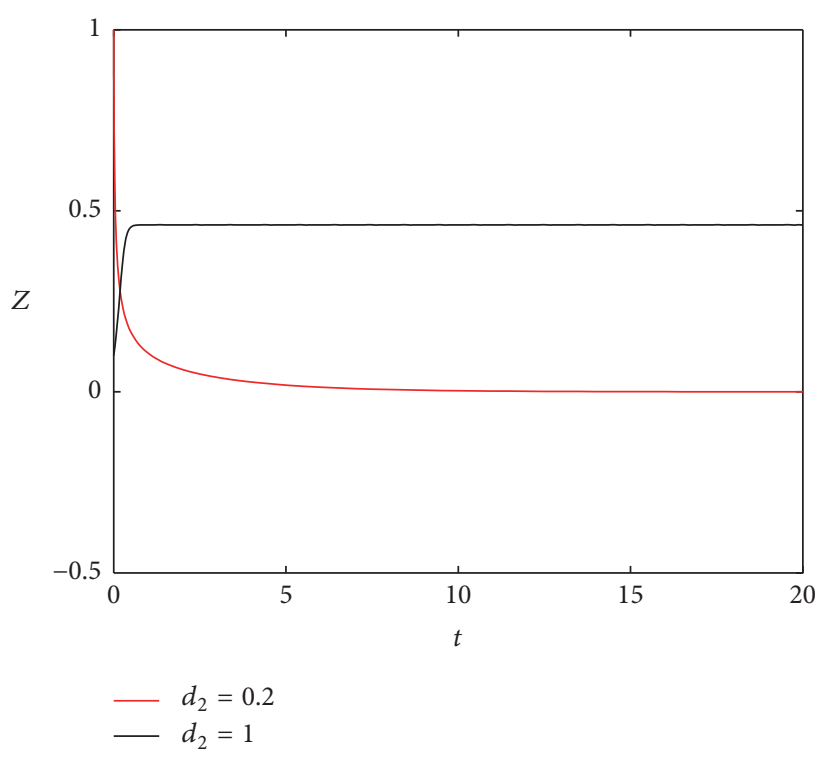

(b)

Figure 1: (a) The bifurcation space diagram. (b) The bifurcation space diagram based on (38) when $\alpha=3, \gamma=1, d_{1}=1, d_{3}=1, \sigma=1 / 2$, and $\beta=2$.

And then we substitute system (28) and (33) into (24) to simplify [32]; we obtain the expressions of the coefficients of $\tau_{0}, g$, and $\mu$ in the Appendix.

And

$$
Z_{i}=\varepsilon W_{i}+\varepsilon^{2} b_{i}+\cdots
$$

So the equation of amplitude is as follows:

$$
\tau_{0} \frac{\partial Z}{\partial t}=\mu Z-g|Z|^{2} Z
$$

Here, we will investigate the dynamics of amplitude equation by using the linear stability analysis $[30,32]$ and study the different pattern. The dynamical systems (38) possess two kinds of solution as follows.

(i) The stationary solution $Z=0$ is stable for $\mu<0$ and unstable for $\mu>0$.

(ii) The solution $Z=\sqrt{\mu / g}$ is only unstable for $\mu<0$.

\section{Simulation}

As the examples of Reaction-Diffusion System with noise, we use the following:

$$
\begin{aligned}
& \frac{\partial u}{\partial t}=f(u, v)+d_{1} \nabla^{2} u+d_{2} R_{1}(u, v) \xi_{1} \\
& \frac{\partial v}{\partial t}=g(u, v)+d_{3} \nabla^{2} u+d_{4} R_{2}(u, v) \xi_{2}
\end{aligned}
$$

where $R_{1}(u, v)=u-u_{0}, R_{2}(u, v)=v-v_{0}, f(u, v)=u-u^{3}-$ $\alpha v-\gamma u v$, and $g(u, v)=u-\beta v$, and obtain the characteristic equation at $\left(u_{0}, v_{0}\right)=(0,0)$. Here, we denote $\alpha=3, \gamma=1$, $d_{1}=1, d_{3}=1$, and $\sigma=1 / 2$ and get the critical value of Hopf bifurcation when $\beta=1+2 d_{2} / \sqrt{2 \pi}+2 d_{4} / \sqrt{2 \pi}$ and Turing bifurcation when $\beta=-2 d_{2} / \sqrt{2 \pi}+2 d_{4} / \sqrt{2 \pi}+2 \sqrt{3}-1$ based on the bifurcation theory in Section 2 .

The model is simulated numerically in two spatial dimensions and employ the zero-flux boundary conditions in (39). We set time step and space step as 0.02 and 1 , respectively. The bifurcation space divide the space into four domains (Figure 1(a)). On bottom domain, locating below two bifurcation spaces, the system lies in the steady state (Figure $3(\mathrm{~d})$ ). The middle domain are regions of pure Turing and pure Hopf in stabilities (Figures 3(b) and 3(c)). On the top, two bifurcation spaces interact (Figure 3(a)). It is found that noise contribute to Turing bifurcation and Hopf bifurcation.

In addition, We find $\tau_{0}=-1.0721, g=-31.5212, \beta_{c}=$ 2.3843, and $\beta=2$ when $d_{2}=0.2, d_{4}=0.1$ and $\tau_{0}=0.1149$, $g=3.5556, \beta_{c}=1.7560$, and $\beta=2$ when $d_{2}=1, d_{4}=0.1$. We get the stability of amplitude equation (Figure $1(\mathrm{~b})$ ) and the corresponding pattern formation (Figures 2(a) and 2(b)). In a nutshell, noise plays an important role in the type of pattern formation and the stability in the system, which provides a new way to investigate the mechanism of pattern formation.

\section{Conclusion}

As we all know, noise could make a bistable system which switches and regulates relevant mechanism [39]. Similarly, it was presented in [40] to understand the biological pattern formation and we presented the spatial pattern with different noise intensities, which gave results supporting that noise could make pattern formation switch (Figures 2(a) and 2(b)) for the Stochastic Reaction-Diffusion Systems. Later some special biological models [21] have been studied and its 


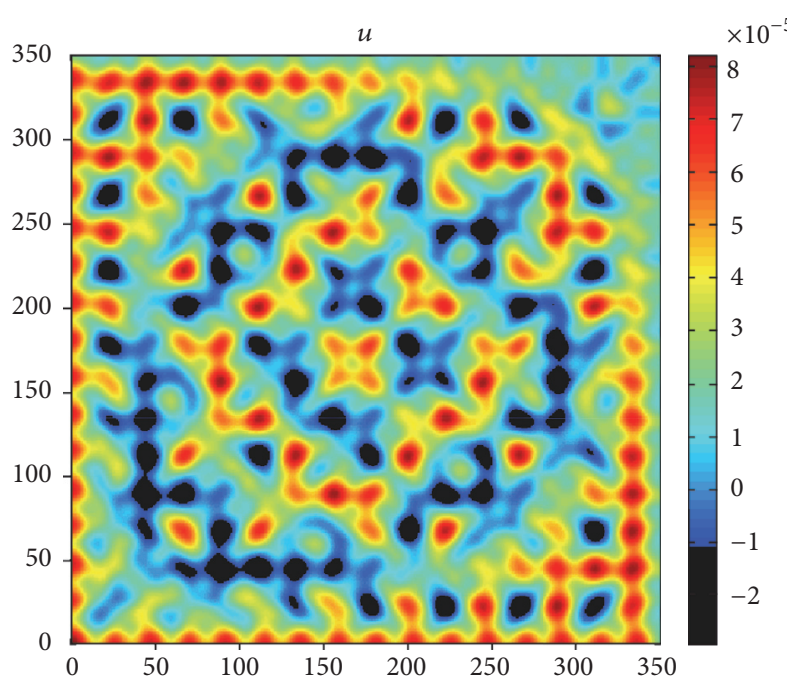

(a)

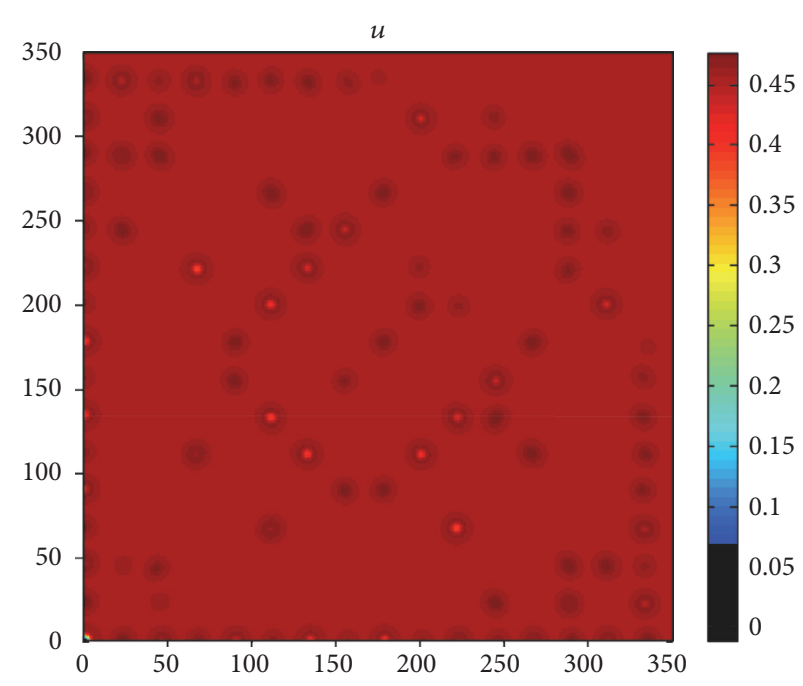

(b)

FIGURE 2: Parameter values and initial perturbation, respectively: $\alpha=3, \gamma=1, d_{1}=1, d_{3}=1, \sigma=1 / 2, d_{4}=0.1, \beta=2$, $\sin (x y)$, and $\cos (x y)$. (a) Pattern formation on the top domain when $d_{2}=0.2$. (b) Pattern formation on the top domain when $d_{2}=1$. Although the amplitude equation is stable, the effect of noise is crucial.

biological mechanism was explained by the type of pattern formation. For example, the system will exhibit a characteristic excursion in phase space before the variables $u$ and $v$ relax back to their rest values [38]. The only spot pattern existing in Figure 2(b) means that the noise exceeds the maximum value that a biological system could bear and makes the biological system worse. Instead, the appropriate noise could keep a biological system working towards better development. For the Turing instability, the different pattern formation occurs in different condition (Figure 3), especially, the pure Turing domain (Figure 3(b)) and the pure Hopf domain (Figure 3(c)), which shows that not only Turing bifurcation but also Hopf bifurcation makes the system vary and decides whether to relax back to their rest values, then keeping good condition. The patterns discussed above show that the distribution and interaction of ion density and electric potential are caused by noise and diffusion. As a result, we can control the distribution of ion by noise, diffusion, and so on. Our research may help cure some diseases caused by the conduction of electrical impulses along a nerve fiber in future.
To summarise, noise effects have been paid much attention due to its strong impact on pattern formation [19-25]. In this article, we presented the theoretical, analytical, and numerical study of the Turing instability accompanied with noise. We examined the effects of noise on pattern formation and the interaction between Hopf bifurcation space and Turing bifurcation space. It is found that the systems with noise effect have rich spatial dynamics by performing a series of numerical simulations. Thus we know that noise plays an important role in Turing bifurcation and Hopf bifurcation. Moreover, we derive the amplitude equation with a pair of wave vector and analyze the stability. It should be noted that noise contribute to the type of pattern formation and the stability. For future study, we would use the theoretical concepts to solve some other problems and find out a general way to deal with it.

\section{Appendix}

$$
\begin{aligned}
N & =\left(\begin{array}{cc}
\gamma u_{1} v_{1} \varepsilon^{2}+\left(\gamma u_{1} v_{2}+\gamma v_{1} u_{2}-u_{1}^{3}-d_{22} u_{1}^{3}\right) \varepsilon^{3}+o\left(\varepsilon^{4}\right) \\
-d_{42} v_{1}^{3} \varepsilon^{3}
\end{array}\right), \\
L_{c} & =\left(\begin{array}{cc}
d_{21}+1+d_{1} \nabla^{2} & -\alpha \\
1 & d_{41}-\beta_{c}+d_{3} \nabla^{2}
\end{array}\right), \\
M & =\left(\begin{array}{ll}
0 & 0 \\
0 & 1
\end{array}\right), \\
N_{1} & =\frac{\partial}{\partial T_{1}}\left(\begin{array}{l}
u_{1} \\
v_{1}
\end{array}\right)-\beta_{1} M\left(\begin{array}{c}
u_{1} \\
v_{1}
\end{array}\right)-\left(\begin{array}{c}
\gamma u_{1} v_{1} \\
0
\end{array}\right),
\end{aligned}
$$




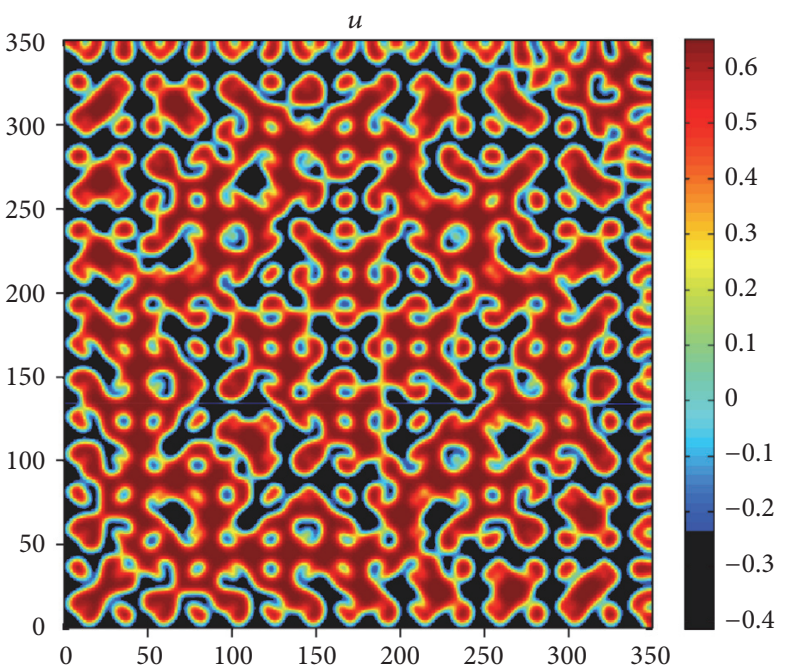

(a)

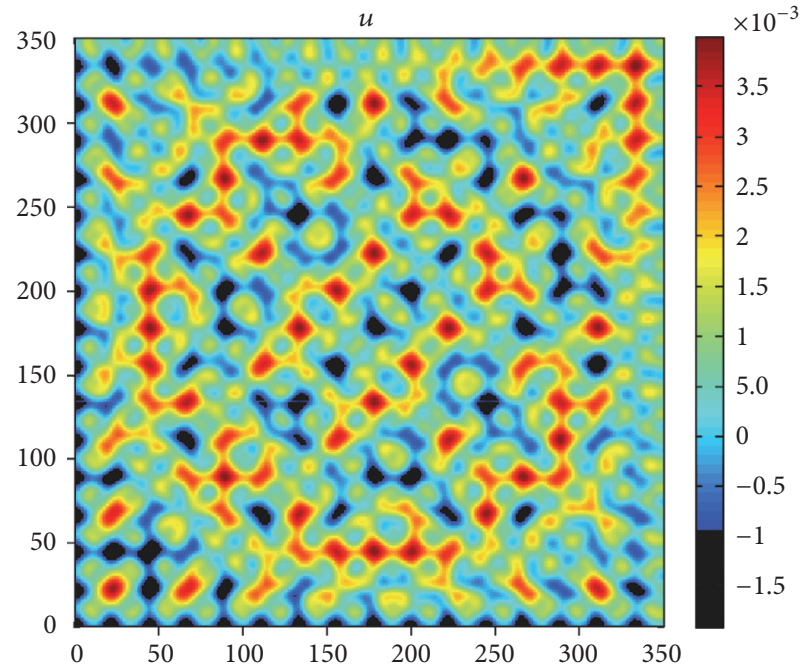

(c)

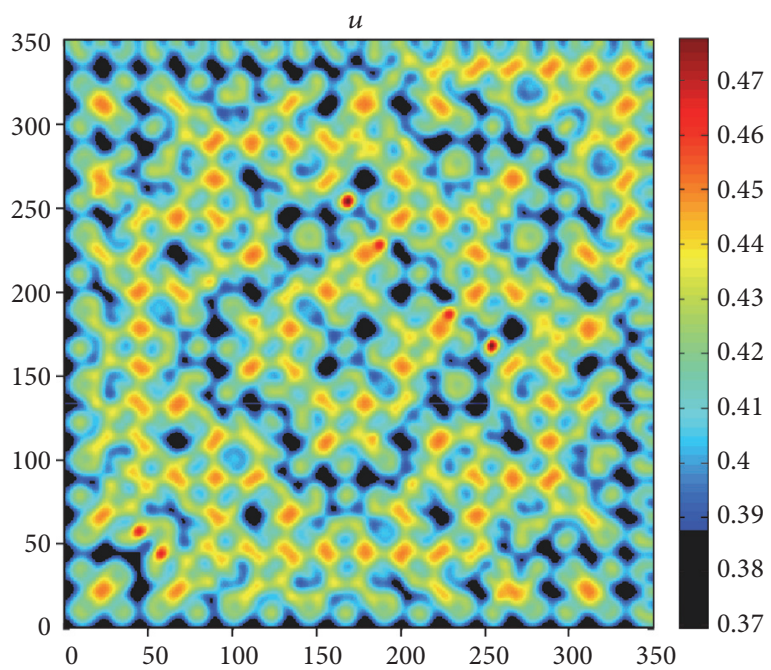

(b)

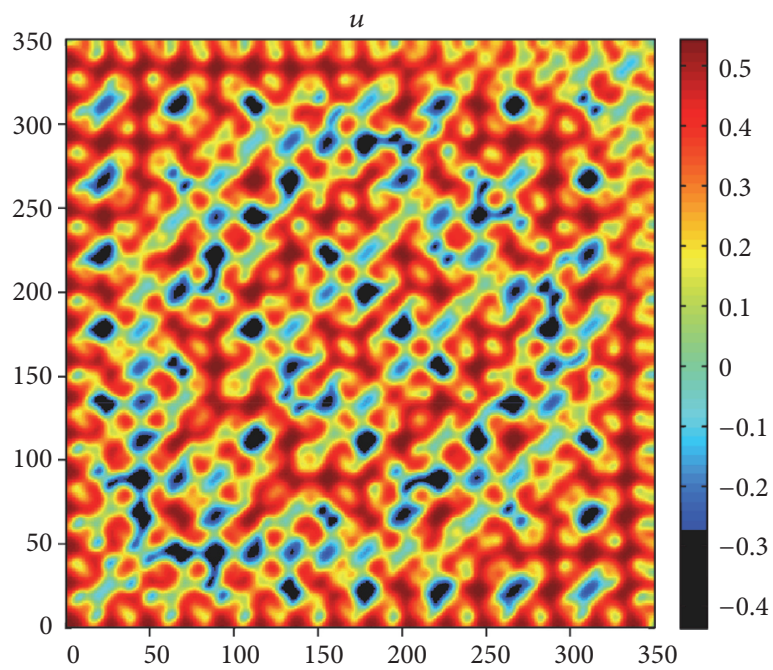

(d)

Figure 3: Parameter values and initial perturbation, respectively: $\alpha=3, \gamma=1, d_{1}=1, d_{3}=1, \sigma=1 / 2, d_{2}=0.1, \sin (x y)$, and $\cos (x y)$. (a) Pattern formation on the top domain when $d_{4}=0.1, \beta=4$. (b) Pattern formation on the right domain when $d_{4}=1$, $\beta=2$. (c) Pattern formation on the left domain when $d_{4}=0.1, \beta=2$. (d) Pattern formation on the bottom domain when $d_{4}=1, \beta=2$.

$$
\begin{aligned}
N_{2} & =\frac{\partial}{\partial T_{1}}\left(\begin{array}{l}
u_{2} \\
v_{2}
\end{array}\right)+\frac{\partial}{\partial T_{2}}\left(\begin{array}{l}
u_{1} \\
v_{1}
\end{array}\right)-\beta_{1} M\left(\begin{array}{l}
u_{2} \\
v_{2}
\end{array}\right)-\beta_{2} M\left(\begin{array}{l}
u_{1} \\
v_{1}
\end{array}\right)\left(\begin{array}{c}
\gamma u_{1} v_{2}+\gamma u_{2} v_{1}-u_{1}^{3}-d_{22} u_{1}^{3} \\
d_{42} v_{1}^{3}
\end{array}\right), \\
x_{1} & =p y_{1} \\
p & =\beta_{c}-d_{41}+d_{3} k_{c}^{2} \\
q & =d_{1} k_{c}^{2}-d_{21}-1 \\
a_{0} & =\left(d_{41}-\beta_{c}\right) b_{0} \\
b_{0} & =\frac{2|Z|^{2} \gamma p}{\left(d_{21}+1\right)\left(d_{41}-\beta_{c}\right)-\alpha} \\
a_{1} & =p b_{1} \\
a_{i i} & =\left(\beta_{c}+4 d_{3} k_{c}^{2}-d_{41}\right) b_{i i}
\end{aligned}
$$




$$
\begin{aligned}
b_{i i} & =\frac{\gamma p|Z|^{2}}{\left(d_{21}+1-4 d_{1} k_{c}^{2}\right)\left(\beta_{c}+4 d_{3} k_{c}^{2}-d_{41}\right)-\alpha}, \\
B & =\left(\begin{array}{l}
\left(d_{21}+1\right) X_{0}-\alpha Y_{0}+\left(\left(d_{21}+1-d_{1} k_{c}^{2}\right) X_{1}-\alpha Y_{1}\right) e^{i k_{c} r}+\left(\left(d_{21}+1-4 d_{1} k_{c}^{2}\right) X_{11}-\alpha Y_{11}\right) e^{i 2 k_{c} r}+c . c \\
X_{0}+\left(-\beta_{c}+d_{41}\right) Y_{0}+\left(X_{1}-\left(\beta_{c}-d_{41}+d_{3} k_{c}^{2}\right) Y_{1}\right) e^{i k_{c} r}+\left(X_{11}-\left(\beta_{c}-d_{41}+4 d_{3} k_{c}^{2}\right) Y_{11}\right) e^{i 2 k_{c} r}+c . c
\end{array}\right) \\
\tau_{0} & =\frac{p+q}{q}, \\
g q & =-\frac{a_{0}+a_{i i}+p b_{0}+p b_{i i}}{|Z|^{2}}+p^{3}+d_{22} p^{3}+d_{42} q \\
\mu & =\beta_{c}-\beta, \\
C & =\frac{\partial}{\partial T_{1}}\left(\begin{array}{c}
u_{1} \\
v_{1}
\end{array}\right)-\beta_{1}\left(\begin{array}{c}
0 \\
Z e^{i k_{c} r}+c . c .
\end{array}\right)-\left(\begin{array}{c}
\gamma u_{1} v_{1} \\
0
\end{array}\right) .
\end{aligned}
$$

Fredholm Solubility Condition. The perturbation equation has $O(1): L u_{0}=0, O\left(\varepsilon^{i}\right): L u_{i}=q_{i}, q_{i}$ which is nonlinear function about $x_{0}, \ldots, x_{i-1}$. In order to remove the resonance term, $q_{i}$ cannot resonate with the nontrivial null space of linear operator $L$, namely, $u_{0}$. Then every linear operator defines an adjoint operator $L^{*}$. The consistency of the solution of equation $L u=q$ requires $u^{+} q=0$, where $L^{*} u^{+}=0, l_{i j}^{*}=\bar{l}_{j i}$. Simply put, Fredholm solubility condition is $u^{+} q=0$.

\section{Competing Interests}

The authors declare that there is no conflict of interests regarding the publication of this paper.

\section{Acknowledgments}

This work is supported by the National Natural Science Foundation of China (11272277, 11572278, and 11572084) and Innovation Scientists and Technicians Troop Construction Projects of Henan Province (2017JR0013).

\section{References}

[1] A. M. Turing, "The chemical basis of morphogenesis," Philosophical Transactions of the Royal Society of London, Series B: Biological Sciences, vol. 237, no. 641, pp. 37-72, 1952.

[2] H. G. Othmer and L. E. Scriven, "Instability and dynamic pattern in cellular networks," Journal of Theoretical Biology, vol. 32, no. 3, pp. 507-537, 1971.

[3] H. Liu and W. Wang, "The amplitude equations of an epidemic model," Science Technology and Engineering, vol. 10, no. 8, pp. 1929-1933, 2010.

[4] E. Ahmed, H. A. Abdusalam, and E. S. Fahmy, "On telegraph coupled map lattice and its applications," International Journal of Modern Physics C, vol. 12, no. 10, pp. 1525-1535, 2001.

[5] Q. Zheng and J. Shen, "Dynamics and pattern formation in a cancer network with diffusion," Communications in Nonlinear Science and Numerical Simulation, vol. 27, no. 1-3, pp. 93-109, 2015.
[6] I. Lee and U. I. Cho, "Pattern formations with Turing and Hopf oscillating pattern," Bulletin of the Korean Chemical Society, vol. 21, no. 12, pp. 1213-1216, 2000.

[7] B. Peña and C. Pérez-García, "Stability of turing patterns in the Brusselator model," Physical Review E. Statistical, Nonlinear, and Soft Matter Physics, vol. 64, no. 5, 2001.

[8] H. Sayama, M. A. de Aguiar, Y. Bar-Yam, and M. Baranger, "Interplay between Turing pattern formation and domain coarsening in spatially extended population models," Forma, vol. 18, no. 1, pp. 19-36, 2003.

[9] P. K. Maini, T. E. Woolley, R. E. Baker, E. A. Gaffney, and S. Seirin Lee, "Turing's model for biological pattern formation and the robustness problem," Interface Focus, vol. 2, no. 4, pp. 487496, 2012.

[10] V. K. Vanag and I. R. Epstein, "Cross-diffusion and pattern formation in reaction-diffusion systems," Physical Chemistry Chemical Physics, vol. 11, no. 6, pp. 897-912, 2009.

[11] D. Fanelli, C. Cianci, and F. Di Patti, "Turing instabilities in reaction-diffusion systems with cross diffusion," The European Physical Journal B, vol. 86, no. 4, article 142, 8 pages, 2013.

[12] J. Shi, Z. Xie, and K. Little, "Cross-diffusion induced instability and stability in reaction-diffusion systems," The Journal of Applied Analysis and Computation, vol. 1, no. 1, pp. 95-119, 2011.

[13] M. Thattai and A. Van Oudenaarden, "Intrinsic noise in gene regulatory networks," Proceedings of the National Academy of Sciences of the United States of America, vol. 98, no. 15, pp. 8614$8619,2001$.

[14] Y. Zhu, J. Shen, and Y. Xu, "Coherence resonance in a noisedriven gene network regulated by small RNA," Theoretical and Applied Mechanics Letters, vol. 4, no. 1, Article ID 013008, 2014.

[15] Y. Zhu, J. Shen, and Y. Xu, "Mechanism of stochastic resonance in a quorum sensing network regulated by small RNAs," Abstract and Applied Analysis, vol. 2013, Article ID 105724, 6 pages, 2013.

[16] M. Viney and S. Reece, "Adaptive noise," Proceedings of the Royal Society of London B, vol. 280, no. 1767, 2013.

[17] S. Scarsoglio, F. Laio, P. D’Odorico, and L. Ridolfi, "Spatial pattern formation induced by Gaussian white noise," Mathematical Biosciences, vol. 229, no. 2, pp. 174-184, 2011. 
[18] Y. Hori and S. Hara, "Noise-induced spatial pattern formation in stochastic reaction-diffusion systems," in Proceedings of the 51st IEEE Conference on Decision and Control (CDC '12), pp. 10531058, Maui, Hawaii, USA, December 2012.

[19] G. Sun, Z. Jin, L. Li, and Q. Liu, "The role of noise in a predatorprey model with Allee effect," Journal of Biological Physics, vol. 35, no. 2, pp. 185-196, 2009.

[20] T. Biancalani, D. Fanelli, and F. Di Patti, "Stochastic turing patterns in the Brusselator model," Physical Review E, vol. 81, no. 4, Article ID 046215, 2010.

[21] A. J. McKane, T. Biancalani, and T. Rogers, "Stochastic pattern formation and spontaneous polarisation: the linear noise approximation and beyond," Bulletin of Mathematical Biology, vol. 76, no. 4, pp. 895-921, 2014.

[22] T. Butler and N. Goldenfeld, "Fluctuation-driven Turing patterns," Physical Review E-Statistical, Nonlinear, and Soft Matter Physics, vol. 84, Article ID 011112, 2011.

[23] Z.-P. Fu, X.-H. Xu, H.-L. Wang, and Q. Ouyang, "Stochastic simulation of turing patterns," Chinese Physics Letters, vol. 25, no. 4, pp. 1220-1223, 2008.

[24] C. Marr and M.-T. Hütt, "Similar impact of topological and dynamic noise on complex patterns," Physics Letters, Section A: General, Atomic and Solid State Physics, vol. 349, no. 5, pp. 302305, 2006.

[25] J.-H. Huh and S. Kai, "Pure noise-induced pattern formations in a nematic liquid crystal," Journal of the Physical Society of Japan, vol. 78, no. 4, Article ID 043601, 2009.

[26] Z. Song, C. Y. Ko, M. Nivala, J. N. Weiss, and Z. Qu, "Calciumvoltage coupling in the genesis of early and delayed afterdepolarizations in cardiac myocytes," Biophysical Journal, vol. 108, no. 8, pp. 1908-1921, 2015.

[27] Z. Song, A. Karma, J. N. Weiss, and Z. Qu, "Long-lasting sparks: multi-metastability and release competition in the calcium release unit network," PLoS Computational Biology, vol. 12, no. 1, Article ID e1004671, 2016.

[28] E. Lau, K. Kossidas, T. Kim et al., "Spatially discordant alternans and arrhythmias in tachypacing-induced cardiac myopathy in transgenic LQT1 rabbits: the importance of I Ks and Ca2+ cycling," PLoS ONE, vol. 10, no. 5, Article ID e0122754, 2015.

[29] W. Wang, H. Liu, Y. Cai, and Z. Li, “Turing pattern selection in a reaction-diffusion epidemic model," Chinese Physics B, vol. 20, no. 7, Article ID 074702, 2011.

[30] Q. Zheng and J. Shen, "Pattern formation in the FitzHughNagumo model," Computers \& Mathematics with Applications, vol. 70, no. 5, pp. 1082-1097, 2015.

[31] A. K. Dutt, "Amplitude equation for a diffusion-reaction system: the reversible Sel'kov model," AIP Advances, vol. 2, no. 4, pp. 124, 2012.

[32] Q. Ouyang, Introduction to Nonlinear Science and Pattern Dynamics, Peking University Press, Beijing, China, 2010.

[33] C. Mayol, R. Toral, and C. R. Mirasso, "Derivation of amplitude equations for nonlinear oscillators subject to arbitrary forcing," Physical Review E-Statistical, Nonlinear, and Soft Matter Physics, vol. 69, no. 6, Article ID 066141, 2004.

[34] J. Murray, Mathematical Biology: I. An Introduction, Interdisciplinary Applied Mathematics, Springer, 2007.

[35] Y. Kuramoto, Chemical Oscillations, Waves and Turbulence, Dover, Mineola, NY, USA, 2003.

[36] G. H. Gunaratne, Q. Ouyang, and H. L. Swinney, "Pattern formation in the presence of symmetries," Physical Review E, vol. 50, no. 4, pp. 2802-2820, 1994.
[37] G.-Q. Sun, L. Li, Z. Jin, Z.-K. Zhang, and T. Zhou, "Pattern dynamics in a spatial predator-prey system with Allee effect," Abstract and Applied Analysis, vol. 2013, Article ID 921879, 12 pages, 2013.

[38] R. FitzHugh, "Impulses and physiological states in theoretical models of nerve membrane," Biophysical Journal, vol. 1, no. 6 , pp. 445-466, 1961.

[39] Y. Xu, Y.-N. Zhu, J. W. Shen, and J. B. Su, "Switch dynamics for stochastic model of genetic toggle switch," Physica A: Statistical Mechanics and Its Applications, vol. 416, pp. 461-466, 2014.

[40] A. T. Winfree, "Varieties of spiral wave behavior: an experimentalist's approach to the theory of excitable media," Chaos. An Interdisciplinary Journal of Nonlinear Science, vol. 1, no. 3, pp. 303-334, 1991. 


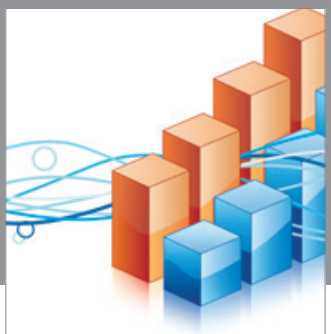

Advances in

Operations Research

vatem alat4

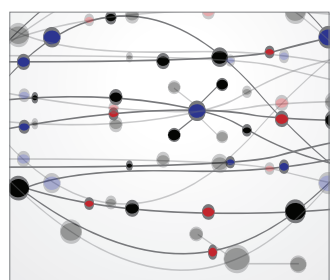

\section{The Scientific} World Journal
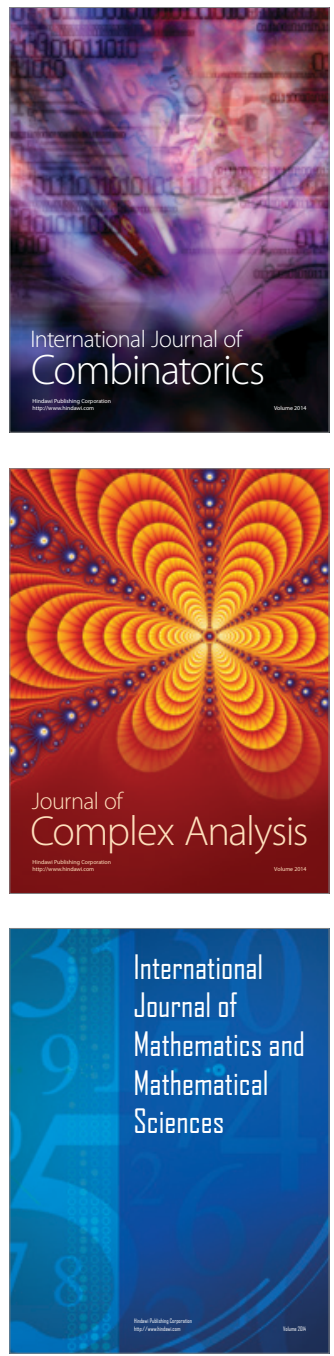
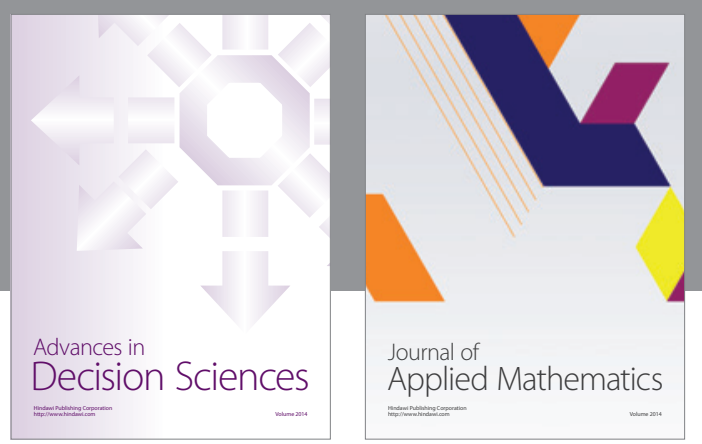

Algebra

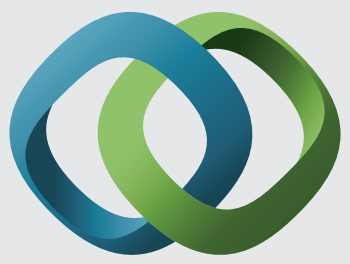

\section{Hindawi}

Submit your manuscripts at

https://www.hindawi.com
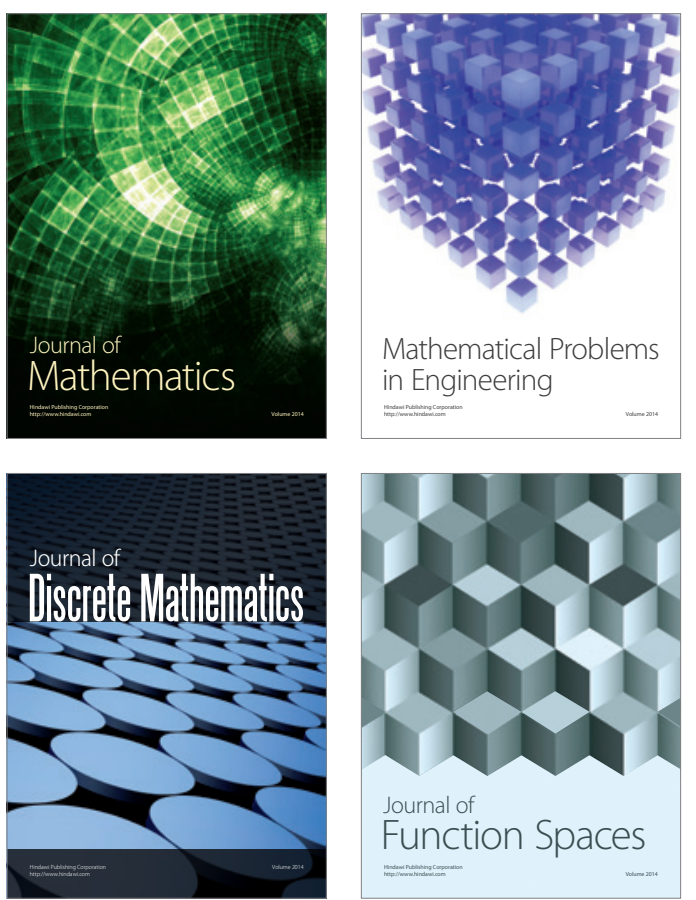

Mathematical Problems in Engineering
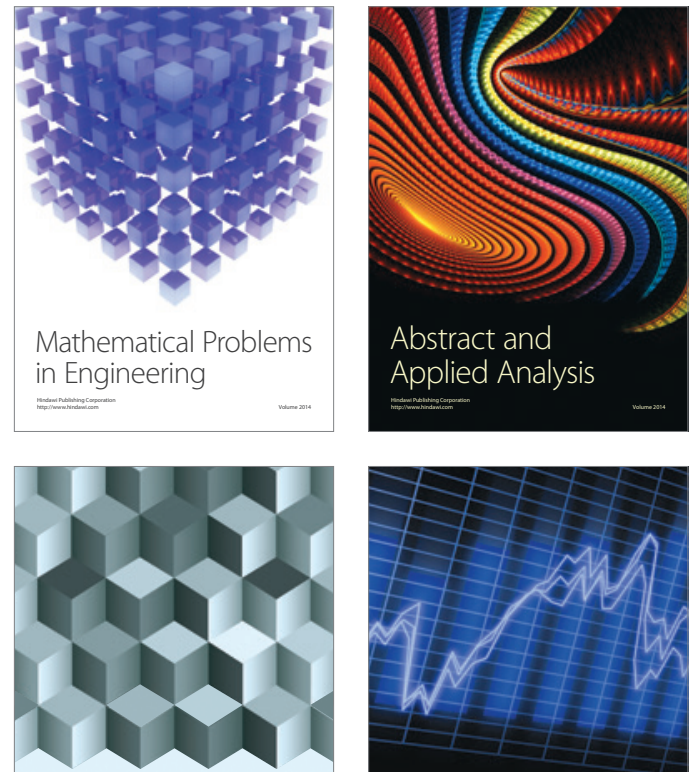

Journal of

Function Spaces

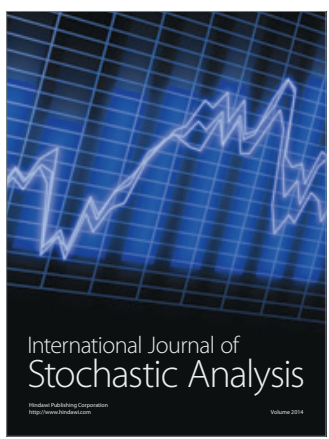

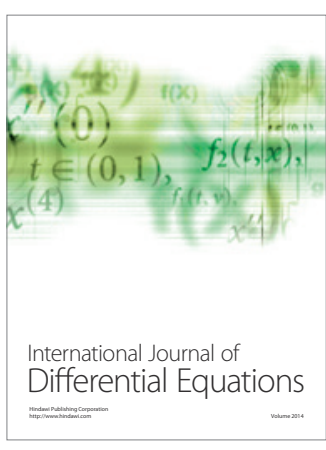
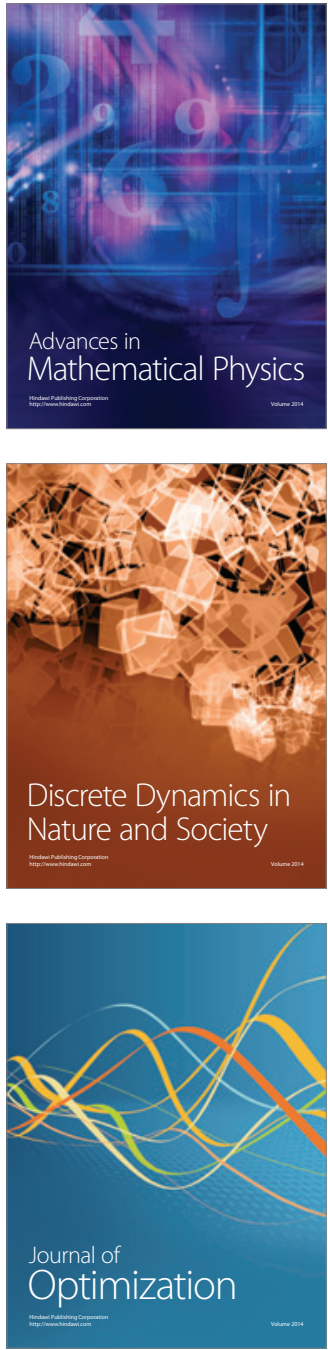